\title{
Deep Inhalation Eliminates Spatial Wrapping
}

\author{
Ahmad Yousef ${ }^{1}$ \\ ${ }^{1}$ School of Computational Science and Engineering, McMaster University, Hamilton, Ontario, Canada \\ *Correspondence: mohamas2@mcmaster.ca
}

\begin{abstract}
This article is to provide evidence that deep inhalation can diminish spatial wrapping. Observation that provide further supporting evidence to our previous hypothesis that assumes inactive 'peripheral brain' can effectively eliminate the wrapping effect.

This might be because the possible forcible reduction in the amount of oxygenated hemoglobin in the brain during the intended inhalation; in turn, different parts in the neocortex might be partially deactivated. Deep exhalations, however, return the perception of warping to its normal state of perception, possibly because of the oxygen-rich blood had returned back to the brain. Deep breathing therefore produces a perceptual rivalry; real perception (deep inhalation) versus illusive perception (deep exhalation).
\end{abstract}

Introduction

Respiratory sinus arrhythmia accommodates the breathing cycles; namely the heart rate raises for the inhalation and declines for exhalation, see references 1 and 2 . Inhalation and exhalation, however, can be cognitively controlled, and these neurophysiological top-down processes can alter the visual motion perception, see reference 3. To date, no studies had done to investigate the effect of deep breathing on stationary illusions such as 'spatial wrapping'; which is a geometrical illusion that not only curves straight lines, but it alters the perception of the centerally-postioned flat rectangle to an illusory paunch, as shown in the central image. In our previous study, we hypothesized that spatial wrapping can be diminished by inactive retinal peripheries, a processed that had been successfully achieved by applying significant reduction against the contrast of the background, see reference 4 . In this study, however, we hypothetically deactivated the 'peripheral brain' itself through forcible significant reduction of oxygen-rich blood in the brain by intended deep inhalation. Deep inhalation seems to respond to our stimulus and produce similar effect of the significant contrast reduction, see reference 4 .
Interestingly, diminishing the temporal asynchronicity between the two conscious brains by deactivating the 'peripheral conscious brain', regardless the technique used, seems to ultimately diminish the spatial wrapping! Noticeably, the central stimulus had the greatest contrast, thus the pupil is assumed to be dilated, see reference 9 and 10; and therefore, the light rays generated by the stimulus should have greater accessibility to the retinal peripheries. These peripheries might signal the brain, but with deep inhalation, the signal collapsed before reaching to its final neurological destinations due to the reduced oxygenated hemoglobin in the brain. In exhalations, however, the oxygen-rich blood returns back to the brain allowing the signal of the retinal peripheries to reach to its destination, and therefore, the perception of spatial

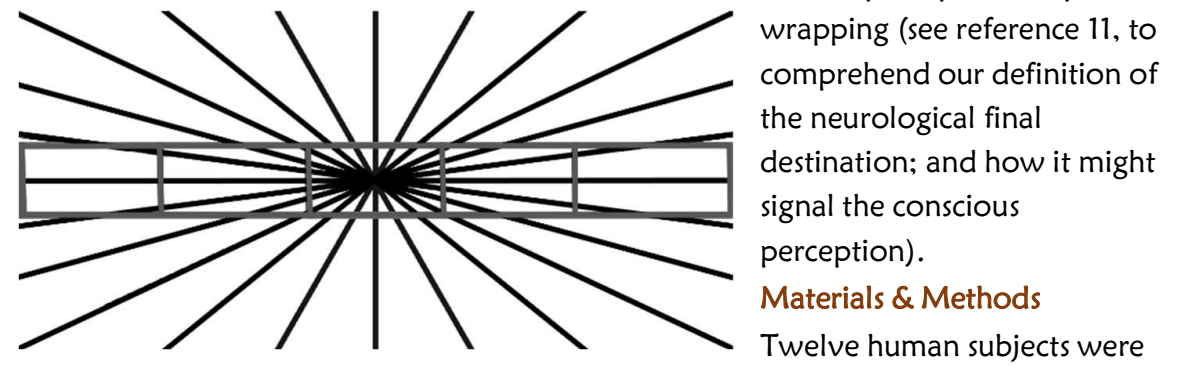

recruited to participate in this study. The experiment stages are as follow, presenting the frame of reference for six seconds to allow the human subjects to comprehend the spatial warping illusion. Afterwards, human subjects has to follow the same 'deep breathing' protocol offered in reference 3 . Our stimulus subtends 8.5 DVA in length, and 4.25 in width. Each subject has to undergo the same experiment, see reference 8, for three times to ensure fine-tuned behavioral data. Each human subject has to quantify the reduction of spatial wrapping at the end of inhalation periods and the end of exhalation periods compared with the frame of reference. To perfectly quantify all of the aspect of spatial wrapping, we had asked the subjects to estimate the wrapping and the 'pop-up' levels of the central rectangle; to be compared with the frame of reference. The behavioral data were collected manually from the three identical trials, and then the three trials values are averaged for each subject separately. The 
aforementioned mean values of all of the twelve subjects are used to estimate generic statistical values, namely, the average and the standard error of the mean.

Results and Conclusion As shown in the up- central infographic, there is a significant reduction in the wrapping perception at the end of the deep inhalation; on another hand, profound exhalation return the illusory wrapping perception mostly back to the reference state. Reduction of oxygen-rich blood in the brain during the deep inhalation seems to play a significant role in deactivating the 'peripheral conscious brain' resulting in diminishing the illusory wrapping perception. Sufficient increase of oxygen-rich blood in the brain during exhalation, however, seems to return the illusory wrapping perception to normal state.

Essential Notification

Readers should have direct access to the stimuli, see reference 12. Advisably, download the movie for the best quality. For the best effect, watch the stimulus with the given DVA values in materials and methods section. Watch the stimulus with dilated pupil to allow the retinal peripheries to work optimally. Advisably, watch the video in moderate environment; namely, fifty percent of the monitor's max. brightness. Caffeine intake also dilate the pupil, in case of pinpoint pupils, see reference 13. During the experiment kindly follow the previously mentioned protocol, namely, take a deep inhalation, execute a profound exhalation, take an extremely very short break, and then repeat the cycle until the end of the trial! We would like to emphasize that we had been relying on the dynamical visual angle, not the static one, see reference 14; however, due to the lack of resources, we are unable to estimate the exact governing equation, thus, we just use the static visual angle in our reports. We generally think that pupil dilates to feed the retinal peripheries, namely, the dilation is not just a cognitive indicator or a reflex, but it may integrate with quantum physics to optimize the human visual awareness generated by unstoppable integrations of 'the central $\&$ the peripheral conscious brains'. Important to attend, all of the stimuli, even those which subtend extremely small static visual angle are able to feed the retinal peripheries due to the unstoppable saccadic eye movements. When we say we deactivated the retinal peripheries; we relies on solid reports that show the peripheries neurophysiological dynamics on elementary stimuli, not tricky or illusive ones. We wish to collaborate with scholars with brain imaging, ECG, and professional eyetracker facilities in challenging investigations. Enquiries may be sent to the author. Finally but interestingly, we have to emphasize that deep breathing had created a perceptual rivalry for a stationary stimulus.

Transactional References

[1] Berntson etal., (1993). Respiratory sinus arrhythmia: autonomic origins, physiological mechanisms, and psychophysiological implications. Psychophysiology.

[2] C. Ludwig, (1847). On the influence of respiratory movements on blood flow in the aortic system. Leipzig.

[3] Yousef, A. 2019. “Deep Breathing Alters Visual Motion Perception.” PsyArXiv. doi:10.31234/osf.io/up3sa.

[4] Yousef, A. 2019. "Idle Retinal Peripheries Diminish Spatial Wrapping." PsyArXiv. doi:10.31234/osf.io/srydk.

[5] Hering, E. (1861). Beitrage zur Physiologie. I. Zur Lehre vom Ortssinne der Netzhaut. Leipzig: Engelmann.

[6] Wundt (1862). Beiträge zur Theorie der Sinneswahrnehmung. Leipzig: Wintersche Verlag.

[7] Holt-Hansen (1961). Hering's illusion. Br. J. Psychol.

[8] Changizi, M. A. (2001). 'Perceiving the present' as a framework for ecological explanations of the misperception of projected angle and angular size. Perception.

[9] Wang , and Munoz. (2014) Modulation of stimulus contrast on the human pupil orienting response, in European Journal of Neuroscience.

[10]Yousef, A. 2019. "Linking Dynamics Between Pupil and Lens." engrXiv. doi:10.31224/osf.io/9c3vk

[11] Yousef, A. 2019. "Consciousness Might Be Localized in Extra Physical Dimensions." PsyArXiv. doi:10.31234/osf.io/angc8.

[12] You may find the stimuli in the following link: https://drive.google.com/drive/folders/1AeKgW_isQqMJXzWfhE sq3_aDMzo8gk-f

[13] Abokyi, etal.,(2017). Caffeine intake is associated with pupil dilation and enhanced accommodation. Eye Journal. [14] Yousef, A. 2019. "The Dynamical Visual Angle." PsyArXiv. doi:10.31234/osf.io/f2kr6. 